Proceedings of the International Symposium on Physics of Materials (ISPMA 14), September 10-15, 2017, Prague

\title{
In Situ Characterization of the Elasticity and Stress-Induced Phase Transformation of NiTi Shape-Memory Alloy
}

\author{
T. Grabec ${ }^{a, *}$, K. Zoubková ${ }^{b}$, P. Stoklasová ${ }^{c}$, M. ŠevČíK ${ }^{c}$, P. Sedlák ${ }^{c}$, M. JanovskÁ ${ }^{c}$, \\ H. SEINER ${ }^{c}$ AND M. LANDA ${ }^{c}$ \\ ${ }^{a}$ Nuclear Physics Institute, Academy of Sciences of the Czech Republic, Řž 130, 25068 Rež, Czech Republic \\ ${ }^{b}$ Faculty of Nuclear Sciences and Physical Engineering, Czech Technical University in Prague, \\ Trojanova 13, 12000 Prague 2, Czech Republic \\ ${ }^{c}$ Institute of Thermomechanics, Academy of Sciences of the Czech Republic, \\ Dolejškova 5, 18200 Prague 8, Czech Republic
}

\begin{abstract}
In the presented paper, a sample of polycrystalline shape-memory $\mathrm{NiTi}$ alloy is studied under compression up to $5 \%$ by the means of laser-excited and laser-detected ultrasound waves. The evolution of a propagation velocity of the surface acoustic wave is measured in situ during mechanical loading. An inverse method based on the Ritz-Rayleigh numerical approach is then used to obtain the development of elastic properties of the sample. This process enables an analysis of the evolution of stress-induced transformation from the austenitic to the martensitic phase with the possibility to describe several stages of such transformation, i.e., the transformation to full R-phase, its reorientation causing strong anisotropy of the polycrystalline sample, and consecutive gradual transition to martensite.
\end{abstract}

DOI: 10.12693/APhysPolA.134.811

PACS/topics: 43.20.+g, 81.05.Bx, 62.20.fg

\section{Introduction}

Elastic properties of single crystals of shape-memory alloys (SMAs) are usually strongly anisotropic [1-3]. Near the transition temperature, the crystal lattice is unstable due to phonon softening [4-6], which increases the anisotropy even further. Laser-ultrasound measurements, such as the resonant ultrasound spectroscopy (RUS) comprise a great tool to study the highly anisotropic materials [7] and the temperature-dependent behavior of materials [8]. Recently, Thomasová et al. [9] presented an ex situ study of the strain-dependence of elastic properties of martensitic structures in polycrystalline NiTi. It was shown in this work that the macroscopic moduli of polycrystalline SMAs can be strongly anisotropic under certain circumstances - in this particular case after stress-induced reorientation of martensitic structure by a uniaxial compression. It is known that those variants of martensite inside the individual grains oriented to the applied stress in an advantageous way grow more than the others [10-12]. It is shown in this study that the in situ measurement offers much more detailed insight into the behavior of the material under stress, with a potential to directly describe the ongoing processes.

The elastic constants of a polycrystalline SMA depend on single-crystal elastic coefficients of the individual

*corresponding author; e-mail: grabec@ujf.cas.cz phases present in the aggregate, the volume fractions of these phases, on the crystallographic texture of austenite, the martensitic microstructure, and the mutual morphology of austenite and martensite [9]. In this work, the development of elastic properties and overall anisotropy of a sample undergoing a compressional loading is studied. The changes in the velocity of the Rayleigh waves during the loading are observed by laser-based ultrasound measurement. To obtain the changes in elasticity coefficients out of the SAW velocities, an inverse procedure utilizing a Ritz-Rayleigh approach as the forward method is presented. It is shown that the originally almost-isotropic compound of R-phase and austenite is transformed to full R-phase, which then reorients, and thus induces strong anisotropy of the sample. During the following loading, the sample undergoes a martensitic phase-transformation. It can be observed from the presented results that the transformation is heterogeneous on microscale. In the last phase of the loading, the stiffening of martensitic phase can be seen, followed by reversible softening during the unloading, thus proving a stress-dependence of elastic properties of the martensite.

\section{Sample and experimental method}

The studied sample was made from a commercially available NiTi with the nominal composition of 50.5 at. $\% \mathrm{Ni}$ and 49.5 at. $\% \mathrm{Ti}$. The sample size is $5 \times 15 \times 50 \mathrm{~mm}^{3}$, one surface of the sample was polished. At room temperature, the material is in a state of austenite and R-phase. The transformation temperatures can be found in [13]. 


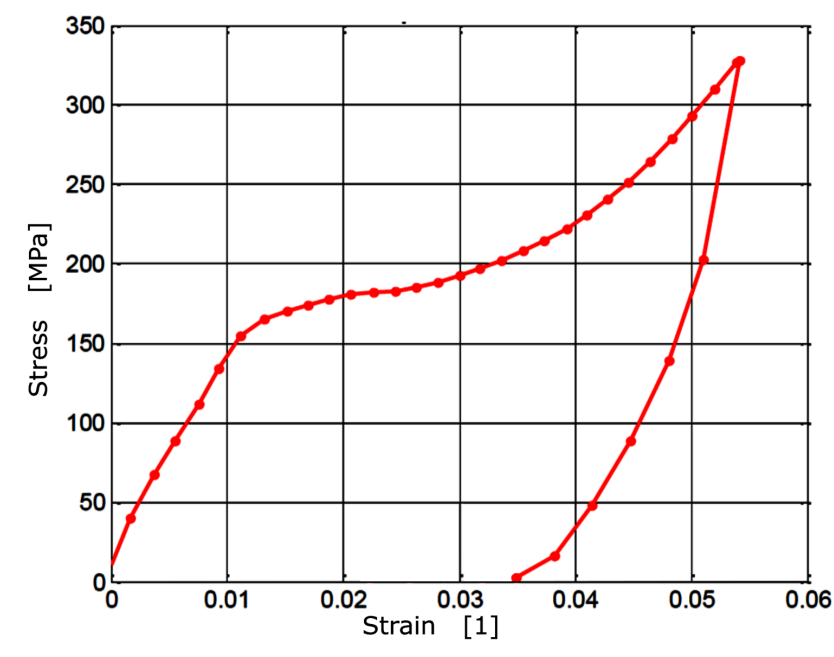

Fig. 1. Stress-strain curve obtained during the measurement (note that the stress is compressional and correspondingly the strain is negative) [14].

A mirror-polished face was used for measurement of the velocity of surface acoustic waves (SAWs). For the excitation, a Nd:YAG laser (1064 nm, 400 ps pulses) focused through a cylindrical lens in order to create a line source was used. The emergent waves were detected by a laser vibrometer $(532 \mathrm{~nm}, \mathrm{cw})$. The sample was mounted on a small in-house built testing machine (maximal load $5 \mathrm{kN}$ ). The testing machine can rotate in order to allow the measurement of the SAW velocity in different directions with respect to the loading. The stress-strain curve of the examined sample at room temperature is shown in Fig. 1. The measurement was conducted in 16 different distances between the excitation and detection spot to obtain the SAW velocity in particular direction. This measurement was made in 5 angles between the propagating wave and loading axis in over 50 strain stages during loading. The resulting evolution of velocities in different directions during the loading is shown in Fig. 2. More details about the experimental setup can be found in [14].

\section{Inverse calculation of the elastic constants}

The experimentally determined SAW velocities were processed by numerical inverse procedure based on the Ritz-Rayleigh approach introduced in [15]:

$$
F\left(c_{i j}\right)=\sum_{p}\left(v_{p}^{\text {cal }}\left(c_{i j}\right)-v_{p}^{\text {exp }}\right)^{2} .
$$

The Ritz-Rayleigh approach offers a fast and robust forward procedure capable of calculation of the velocity of SAW propagating in any direction through a generally anisotropic material with the elastic given. The approach is based on Hamilton's principle, according to which the resonant frequencies and the corresponding modal shape can be found as stationary points of a Lagrangian energy of a harmonically vibrating body, i.e. a computational domain with the constraints chosen in a way to suit the problem solved. To find these stationary points, a discretization of the displacement field into the Legendre polynomials is considered. The solution then contains both bulk and surface resonances of the domain, which can be easily distinguished, e.g. based on the modal shape. The inverse procedure is then based on an iterative process of changing the initial set of elastic constants. The set of corresponding SAW velocities obtained from the forward procedure is then compared to the experimental value via the objective function where $p$ goes through all measured angles, and the superscripts exp and cal denote the calculated and experimental velocity, respectively. For the minimization of this function, a gradient Levenberg-Marquardt method is used. The numerical inverse procedure depicted briefly here was described in more detail by Stoklasová et al. [15]. It was shown that in addition to the spatial dispersion calculated in this case, the method can be modified for calculations of a frequency dispersion in a layered system [16].

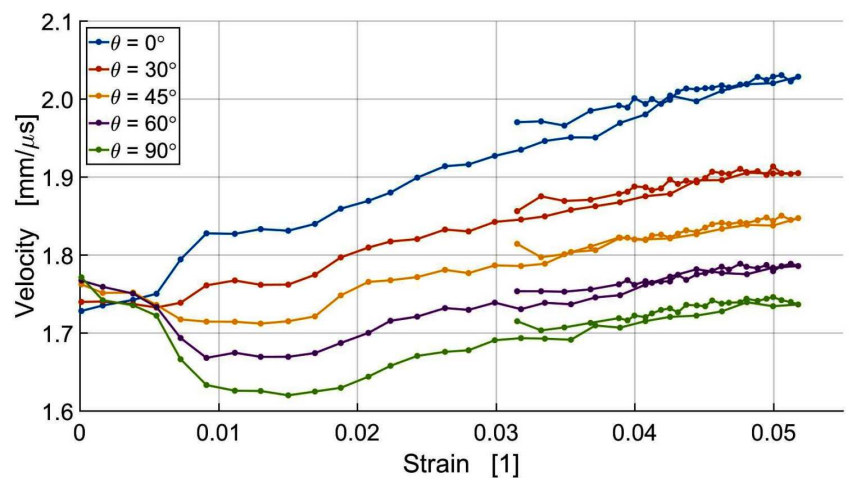

Fig. 2. Experimentally obtained SAW velocities in different angles with respect to strain.

The result of the inverse procedure is a set of elastic constants minimizing the objective function. Considering the nature of the experiment, i.e. the initial isotropy and uniaxial loading, the material is assumed to adopt the transversely isotropic character. As such, it exhibits hexagonal symmetry with 5 independent elastic constants: $c_{11}, c_{33}, c_{13}, c_{44}$, and $c_{66}$. Considering the geometry of the experiment, the direction of the symmetry is assumed to be aligned with $x_{3}$ axis. This set was calculated for each stress level. The error of this set can be estimated by an error-analysis procedure described in detail in Ref. [7]. However, given the informative character of the results presented in this paper, the error is not calculated here. The mass density taken for the calculations was $6.45 \mathrm{~g} / \mathrm{cm}^{3}$.

\section{Results}

Experimental stress-strain curve (Fig. 1) shows a character typical for memory alloys under compression [17]. It shows the initial almost-linear response, then the plateau of martensitic transition between around $1.3 \%$ and $3.5 \%$, and then the following loading of martensite. The measured velocities of SAWs (Fig. 2) and corresponding shear elastic moduli (Fig. 3) document arguably rather peculiar behavior of the material during this compression test. 
In the initial phase of loading up to the start of the martensitic phase transition above $1.3 \%$, a surprisingly strong anisotropy of the sample arises. With a maximum at the beginning of the phase transition, the anisotropy peaks a ratio of 1.35 between the shear elastic moduli. This is believed to be caused by the induction of the reorientation of the trigonal $\mathrm{R}$-phase, and it was checked that this process is fully reversible and can be observed only during in situ measurement under applied stress.

The second part shows the development of elastic constants during the stress-induced martensitic phase transformation. The material stiffens in all directions, as the elastic constants of martensite are higher than the ones of $\mathrm{R}$-phase. However, there is an interesting observation in the character of the progress - whereas $c_{44}$ increases linearly, the $c_{66}$-evolution shows a certain curvature. From this fact, it can be assumed that whereas on a macroscopic level, the martensitic transition in compression proceeds homogeneously, it is heterogeneous on the level of microstructure. The data obtained by SAWs are unfortunately insufficient to obtain a full understanding of the heterogeneity and the process itself, as they mostly carry the information about the shear behavior, and thus must be accompanied by supplement measurements of the longitudinal wave.

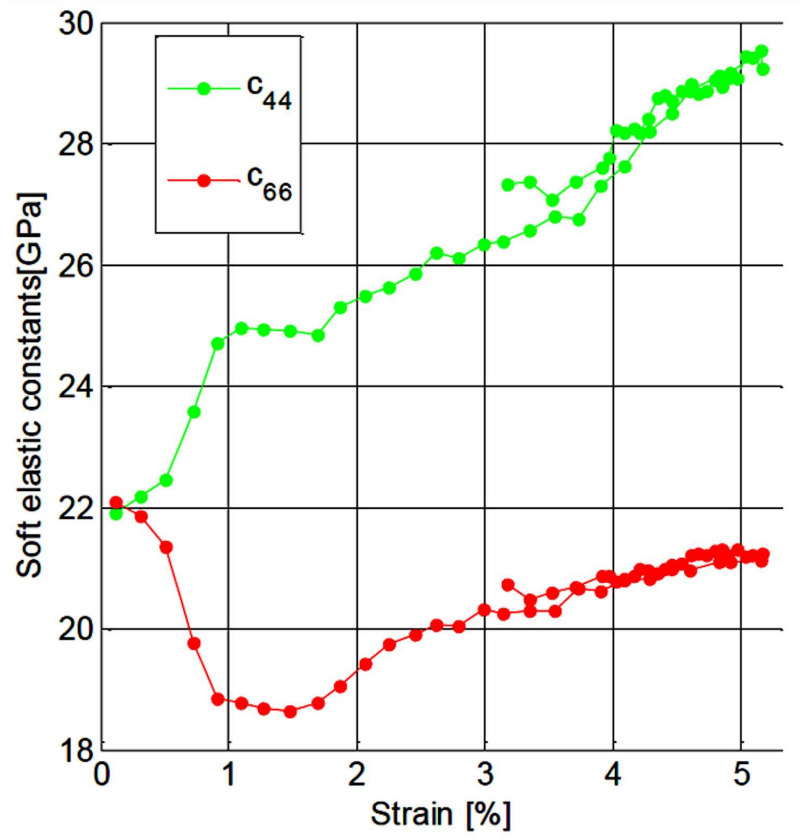

Fig. 3. Resulting shear elastic moduli obtained by the inverse procedure.

The third and last part of the stress-strain curve shows the final loading of a dominantly martensitic sample. It was observed that the elastic coefficients of martensite depend on stress in a fully reversible manner. After unloading, the oriented martensite retains its anisotropic elasticity, which is entirely in compliance with the results found by ex situ measurement presented by Thomasová et al. [9].

\section{Conclusions}

The contribution presented illustrates the evolution of elastic properties of polycrystalline NiTi undergoing a compressional loading, observed in situ by the laser-based ultrasound method focused on surface acoustic waves. It is shown that the in situ measurement has great potential to increase the understanding of the processes in the material in such a case.

The measurement of SAW velocities and their spatial distribution can be realized during the loading. Velocities obtained are then used to determine the development of elasticity coefficients by the inverse procedure based on the Ritz-Rayleigh approach. Since SAWs carry mostly information about the shear behavior, only the shear constants $c_{44}$ and $c_{66}$ can be determined with sufficient accuracy.

Based on the development of the identified elastic constants, several remarks on the stress-dependent behavior of the material can be made. Firstly, the reorientation of $\mathrm{R}$-phase introduces a surprisingly large anisotropy to the sample. The character of the anisotropy remains similar even after the full martensitic phase-transformation. However, the development of the constants is uneven, suggesting a heterogeneous character of transition between the R-phase and martensite. Unfortunately, the presented measurement does not offer sufficient data to completely reveal this character and supplementary experimental data are necessary. One of the possibilities is to combine the SAW with a pulse-echo measurement during the loading.

\section{Acknowledgments}

This work has been supported by OP RDE, MEYS, under the project ESS - SCANDINAVIA - CZ - PROJECT CZ.02.1.01/0.0/0.0/16 013/0001794, and the CSF project of the Research Center AdMAT (14$36566 \mathrm{G})$.

\section{References}

[1] T.M. Brill, S. Mittelbach, W. Assmus, M. Mullner, B. Luthi, J. Phys. Condens. Matter 3, 9621 (1991).

[2] P. Sedlák, H. Seiner, M. Landa, V. Novák, P. Šittner, L. Manosa, Acta Mater. 53, 3643 (2005).

[3] A. González-Comas, L. Manosa, A. Planes, F.C. Lovey, J.L. Pelegrina, C. Guénin, Phys. Rev. B 56, 5200 (1997).

[4] H. Seiner, P. Stoklasová, P. Sedlák, M. Ševčík, M. Janovská, M. Landa, T. Fukuda, T. Yamaguchi, T. Kakeshita, Acta Mater. 105, 182 (2016).

[5] A. Planes, Ll. Manosa, Solid State Phys. 55, 159 (2001).

[6] H. Seiner, L. Bicanová, P. Sedlák, M. Landa, L. Heller, I. Aaltio, Mater. Sci. Eng. A 521, 205 (2009).

[7] P. Sedlák, H. Seiner, J. Zídek, M. Janovská, M. Landa, Exp. Mech. 54, 1073 (2014).

[8] M. Landa, P. Sedlák, P. Šittner, H. Seiner, V. Novák, Mater. Sci. Eng. A 462, 320 (2007). 
[9] M. Thomasová, H. Seiner, P. Sedlák, M. Frost, M. Sevčík, I. Szurman, R. Kocich, J. Drahokoupil, P. Sittner, M. Landa, Acta Mater. 123, 145 (2017).

[10] P. Šittner, D. Neov, P. Lukáš, D.M. Toebbens, J. Neutron Res. 12, 15 (2004).

[11] H.-R. Wenk, I. Lonardelli, D. Williams, Acta Mater. 52, 1899 (2004).

[12] P. Šittner, P. Lukáš, V. Novák, M.R. Daymond, G.M. Swallowe, Mater. Sci. Eng. A 378, 97 (2004).

[13] P. Šittner, M. Landa, P. Lukáš, V. Novák, Mech. Mater. 38, 475 (2006).
[14] M. Ševčík, T. Grabec, P. Stoklasová, M. Janovská, K. Zoubková, P. Sedlák, H. Seiner, M. Landa, Acta Phys. Pol. A 134, 807 (2018).

[15] P. Stoklasová, P. Sedlák, H. Seiner, M. Landa, Ultrasonics 56, 381 (2015).

[16] T. Grabec, P. Sedlák, P. Stoklasová, M. Thomasová, D. Shilo, M. Kabla, H. Seiner, M. Landa, Smart. Mater. Struct. 25, 127002 (2016).

[17] P. Sittner, L. Heller, J. Pilch, C. Curfs, T. Alonso, D. Favier, J. Mater. Eng. Perform. 23, 2303 (2014). 